Rev. Elev. Méd. Vét. Pays trop., 1976, 29 (1) : 17-22

\title{
Biométrie, morphologie et virulence de Trypanosoma (Nannomonas) congolense à travers 640 passages sur souris en 10 ans
}

\author{
par S. M. TOURE (*) \\ (avec la collaboration technique de B. KEBE, M. SEYE et N. BA)
}

\begin{abstract}
RÉSUMÉ
3 souches de Trypanosoma congolense ont été récoltées en 1964 dans le sud-ouest du Sénégal et l'une d'elles passée sur souris pendant plus de 10 ans. Les 3 souches présentent la variation Dimorphon. Au cours des passages sur souris, on assiste à un allongement de la longueur des Trypanosomes. Le fait de réaliser des passages élevés sur souris, même pendant 10 ans, n'entraîne pas une perte de virulence de $T$. congolense à l'égard des bovins et il semble illusoire d'obtenır, par le procédé des passages, des souches utilisables pour vacciner.
\end{abstract}

\section{INTRODUCTION}

Trois souches de Trypanosomes, de l'espèce Trypanosoma (Nannomonas) congolense, ont été isolées en août 1964 dans deux villages du Sénégal (Djilor et Keur Baba Diouf, Région du SineSaloum, $14^{\circ}$ nord $16^{\circ} 20^{\prime}$ ouest) et passées sur souris. Deux des souches, dénommées respectivement $\mathrm{C}_{2} \mathrm{~T}$ et $\mathrm{C}_{2} \mathrm{Q}$, proviennent de deux bovins et la troisième, $C_{3} T Q$, d'un âne. Les villages d'origine sont situés à côté d'une forêt infestée de glossines (Glossina morsitans submorsitans) et non loin de marigots à $G$. palpalis gambiensis.

Au cours des passages successifs, deux des souches ont été perdues $\left(C_{2} Q\right.$ en 1965 après 30 passages et $C_{3}$ TQ en 1970 après 367 passages) et la troisième a pu être maintenue jusqu'à présent sur souris (704\% passage en mai 1975). La présente note a pour objet l'étude biométrique de Trypanosoma congolense à travers les passages sur souris, la morphologie et ses variations ainsi que la virulence pour les bovins après dix années de passages.

(*) I. S. R. A., Laboratoire national de l'Elevage et de Recherches vétérinaires, B. P. 2057, Dakar, Sénégal.

\section{I. ÉTUDE BIOMÉTRIQUE DES SOUCHES A TRAVERS LES PASSAGES}

\section{Premières semaines de passage}

La détermination spécifique des Trypanosomes est fondée sur ces premiers passages. En effet, les lames faites sur le terrain à partir des bovins et de l'âne n'avaient pas les qualités requises pour une étude biométrique.

Les observations microscopiques, faites au cours de ces passages, montrent des formes de grande taille en comparaison avec d'autres, de petite taille, souvent larges, ce qui pouvait faire penser a priori à une population hétérogène de Trypanosomes. Cependant, l'analyse biométrique ne reflète pas cette hétérogénéité car on obtient une courbe de Gauss assez régulière. Six populations ont été mesurées, soit :

$C_{2} \mathrm{~T}:\left\{\begin{array}{l}386 \text { Trypanosomes de } 1^{\mathrm{er}} \text { passage, } \\ 219 \text { Trypanosomes de } 3^{\mathrm{e}} \text { passage. }\end{array}\right.$
$\mathrm{C}_{2} \mathrm{Q}:\left\{\begin{array}{l}229 \text { Trypanosomes de } 1^{\mathrm{er}} \text { passage, } \\ 166 \text { Trypanosomes de } 4^{\mathrm{e}} \text { passage. }\end{array}\right.$
$\mathrm{C}_{3} \mathrm{TQ}:\left\{\begin{array}{l}145 \text { Trypanosomes de } 1^{\mathrm{er}} \text { passage, } \\ 236 \text { Trypanosomes de } 3^{\mathrm{e}} \text { passage. }\end{array}\right.$ 
La longueur moyenne pour les six populations oscille entre $13,34 \pm 0,16 \mu \mathrm{m}$ et $14,26 \pm 0,14 \mu \mathrm{m}$. Les moyennes observées sont, dans loordre croissant : $13,34 \pm 0,16 \mu \mathrm{m} ; 13,38 \pm 0,11 \mu \mathrm{m}$; $13,86 \pm 0,11 \mu \mathrm{m} ; 13,90 \pm 0,13 \mu \mathrm{m}$; $14,03 \pm 0,21 \mu \mathrm{m}$ et $14,26 \pm 0,14 \mu \mathrm{m}$.

Par commodité d'appréciation qualitative, il est convenu ici de considérer comme Trypanosomes de taille moyenne tous ceux dont la longueur est comprise entre $13,00 \mu \mathrm{m}$ et $14,60 \mu \mathrm{m}$, c'est-à-dire un intervalle de classe en deçà de la moyenne la plus faible et à un intervalle de classe au-delà de la moyenne la plus élevée. Ainsi, les Trypanosomes considérés comme courts ne dépassent pas $13,00 \mu \mathrm{m}$ tandis que les formes longues commencent à partir de $14,60 \mu \mathrm{m}$. Le tableau qui suit (tableau $n^{\circ} \mathrm{I}$ ) résume les données numériques de l'analyse selon cette convention. Il y a lieu d'insister sur le fait que cette division en trois séries n'a pas du tout la même signification que pour les Trypanosomes du sous-genre Trypanozoon et que nous ne l'avons adoptée que pour illustrer la tendance à l'allongement à travers les passages.
L'étude critique des résultats de la biométrie dans ces premiers passages montre que ces souches de Trypanosoma congolense présentent une variation de type Dimorphon. Il apparaît toutefois que les moyennes réelles que nous avons obtenues $(13,34$ à $14,26 \mu \mathrm{m})$ ainsi que les valeurs adoptées à un intervalle de classe de ces moyennes, soit de 13 à $14,60 \mu \mathrm{m}$, caractérisent une variation de type intermédiaire, si l'on compare les données avec celles indiquées par HOARE, 1972 (10) se référant à GODFREY, 1960 (5) et FAIRBAIRN, 1962 (3). Notre adhésion à une variation de type Dimorphon tient au pourcentage particulièrement élevé de formes longues, 29 à $46 \mathrm{p}$. 100 , de très loin supérieur aux 11 p. 100 indiqués pour la variation de type intermédiaire et cela même en adoptant comme point de départ des formes longues $14,60 \mu \mathrm{m}$ au lieu de $14,26 \mu \mathrm{m}$.

\section{Sixième mois de passage et années suivantes}

$\mathrm{Au}$ sixième mois, la biométrie des $22^{\mathrm{e}}$ et $24^{\mathrm{e}}$ passages conduit aux résultats indiqués dans le tableau $\mathrm{n}^{\circ}$ II.

TABLEAU $\mathrm{N}^{\circ}$ I-Analyse biométrıque dans les premiers passages

\begin{tabular}{|c|c|c|c|c|c|c|c|}
\hline \multicolumn{2}{|c|}{ Souches } & $\left\{\begin{array}{l}\mathrm{C}_{2} \mathrm{~T} \\
\text { ler } \\
\text { passage }\end{array}\right.$ & $\stackrel{\mathrm{C}_{2} \mathrm{Q}}{\text { ler passage }}$ & $\begin{array}{c}\mathrm{C}_{3} \text { IQ } \\
\text { 1er passage }\end{array}$ & $3 e^{C_{2}} \stackrel{T}{\text { passage }}$ & $\begin{array}{c}\mathrm{C}_{2} \mathrm{Q} \\
4 \mathrm{e} \text { passage }\end{array}$ & $\begin{array}{c}\mathrm{C}_{3} \mathrm{TQ} \\
3 \mathrm{e} \text { passage }\end{array}$ \\
\hline \multicolumn{2}{|c|}{$\begin{array}{l}\text { Nombre de trypanosomes } \\
\text { mesurés }\end{array}$} & 386 & 229 & 145 & 219 & 166 & 236 \\
\hline \multicolumn{2}{|c|}{$\begin{array}{l}\text { Amplitude de la } \\
\text { variation (en } \mu \mathrm{m})\end{array}$} & $8 \quad-19,10$ & $9,60-18,70$ & $10-18,70$ & $10,40-17,90$ & $9,20-18,70$ & $8,80-18,30$ \\
\hline \multicolumn{2}{|c|}{$\begin{array}{l}\text { Longueur moyenne } \\
(\mathrm{en} \mu \mathrm{m})\end{array}$} & 13,34 & 14,03 & 14,26 & 13,86 & 13,90 & 13,38 \\
\hline \multicolumn{2}{|c|}{$\begin{array}{l}\text { Erreur sur la moyenne } \\
\text { (en } \mu \text { ni })\end{array}$} & $\pm 0,16$ & $\pm 0,21$ & $\pm 0,14$ & $\pm 0,11$ & $\pm 0,13$ & $\pm 0,11$ \\
\hline \multirow{3}{*}{$\begin{array}{l}\text { Formes de } \\
\text { petite } \\
\text { tallle }\end{array}$} & $\begin{array}{l}\text { Intervalle } \\
(\mu \mathrm{m})\end{array}$ & $8-13,00$ & $9,60-13,00$ & $10 \quad-13,00$ & $10,40-13,00$ & $9,20-13,00$ & $8,80-13,00$ \\
\hline & $\begin{array}{l}\text { Nombre de } \\
\text { tryp. }\end{array}$ & 98 & 43 & 20 & 40 & 29 & 54 \\
\hline & Pourcentage & 25 & 18 & 14 & 19 & 17 & 23 \\
\hline \multirow{3}{*}{$\begin{array}{l}\text { Formes de } \\
\text { taille } \\
\text { inter- } \\
\text { mêdiaire }\end{array}$} & $\begin{array}{l}\text { Intervalle } \\
(\mu \mathrm{m})\end{array}$ & $13,00-14,60$ & $13,00-14,60$ & $13,00-14,60$ & $13,00-14,60$ & $13,00-14,60$ & $13,00-14,60$ \\
\hline & $\begin{array}{l}\text { Nombre de } \\
\text { tryp. }\end{array}$ & 178 & 87 & 58 & 89 & 73 & 114 \\
\hline & Paurcentage & 46 & 38 & 40 & 41 & 44 & 48 \\
\hline \multirow{3}{*}{$\begin{array}{l}\text { Formes de } \\
\text { grande } \\
\text { taille }\end{array}$} & $\begin{array}{l}\text { Intervalle } \\
(\mu \mathrm{m})\end{array}$ & $14,60-19,10$ & $14,60-18,70$ & $14,60-18,70$ & $14,60-17,90$ & $14,60-18,70$ & $14,60-18,30$ \\
\hline & $\begin{array}{l}\text { Nombre de } \\
\text { tryp. }\end{array}$ & 110 & 101 & 67 & $8 B$ & 64 & 68 \\
\hline & Pourcentage & 29 & 44 & 46 & 40 & 39 & 29 \\
\hline
\end{tabular}


TABLEAU $N^{\circ}$ II-Analyse biométrique dans les 22 e et 24 e pasagas.

\begin{tabular}{|l|c|c|c|}
\hline \multicolumn{1}{|c|}{ Souches } & $\mathrm{C}_{2} \mathrm{~T}$ & $\mathrm{C}_{2} \mathrm{Q}$ & $\mathrm{C}_{3}$ TQ \\
\hline Nombre de trypanosomes mesurés & 200 & 200 & 200 \\
\hline Amplitude (en $\mu \mathrm{m})$ & $8,80-17,90$ & $10-17,30$ & $9,60-18,40$ \\
\hline Longueur moyenne $(\mu \mathrm{m})$ & 13,12 & 13,07 & 14,16 \\
\hline Erreur sur la moyenne $(\mu \mathrm{m})$ & $\pm 0,13$ & $\pm 0,16$ & $\pm 0,20$ \\
\hline
\end{tabular}

TABLEAU N ${ }^{\circ}$ III-C 2 T, de 1964 à 1974 .

\begin{tabular}{|c|c|c|c|c|c|}
\hline $\begin{array}{r}\mathrm{N}^{\circ} \text { de } \\
\text { passage }\end{array}$ & Date & $\begin{array}{c}\text { Nombre de } \\
\text { trypanosomes }\end{array}$ & Amplitude $(\mu \mathrm{m})$ & $\begin{array}{l}\text { Longueur } \\
\text { moyenne (m) }\end{array}$ & Erreur $(\mu \mathrm{m})$ \\
\hline ler & Septembre 1964 & 386 & $8-19,10$ & 13,34 & $\pm 0,16$ \\
\hline $3 e$ & Novembre 1964 & 219 & $10,40-17,90$ & 13,86 & $\pm 0,11$ \\
\hline $22 e$ & Mars 1965 & 200 & $10,80-17,90$ & 13,12 & $\pm 0,13$ \\
\hline $163 e$ & Mars 1967 & 100 & $11,87-20,62$ & 16,67 & $\pm 0,19$ \\
\hline $494 \mathrm{e}$ & Avril 1972 & 100 & $12,35-19,47$ & 16,08 & $\pm 0,24$ \\
\hline $605 e$ & Novembre 1973 & 100 & $12,35-20,90$ & 16,08 & $\pm 0,24$ \\
\hline $640 \mathrm{e}$ & Mai 1974 & 210 & $10,50-19,66$ & 14,80 & $\pm 0,17$ \\
\hline
\end{tabular}

Les différentes souches ne présentent aucun changement significatif au cours des 6 premiers mois de passage.

C'est au cours des années suivantes qu'il a été constaté un allongement appréciable de la longueur moyenne des Trypanosomes de la souche $\mathrm{C}_{2} \mathrm{~T}$ comme en témoigne le tableau III.

Les passages élevés font apparaître une très nette modification de cette souche dans le sens de l'allongement de la longueur des Trypanosomes. $\mathrm{Du} 494^{\circ}$ au $640^{\circ}$ passage, les trois séries de mensurations effectuées donnent des moyennes de $16,08 \mu \mathrm{m} \pm 0,24$ et $14,80 \mu \mathrm{m} \pm 0,17$ qui sont nettement supérieures aux valeurs des premiers passages : 82 p. 100 et 74 p. 100 respectivement au $494^{\circ}$ et au $605^{\circ}$ passage mesurent plus de $15 \mu \mathrm{m}$; mais le pourcentage n'est que de 39,5 au $640^{\circ}$ passage, ce qui laisse apparaître la probabilité de fluctuations selon les passages.

\section{II. ÉTUDE MORPHOLOGIQUE COMPARÉE}

Comme signalé plus haut, c'est plus sur la morphologie que repose l'aspect hétérogène des populations dans les premiers passages. La varia- tion concerne la largeur des Trypanosomes et la forme particulière, la position et le nombre des organites intracytoplasmiques. A l'intérieur des trois catégories définies ci-dessus, à savoir : taille intermédiaire, grande taille et petite taille, on rencontre des formes massives dont la largeur atteint souvent $5 \mu \mathrm{m}$ au lieu de 1,5 à $2 \mu \mathrm{m}$ comme c'est habituel, et qui ne sont pas des formes de division. La plupart de ces formes ont l'extrémité postérieure obtuse ; le kinétoplaste est plus petit que dans les formes normales, punctiforme, subterminal, plus souvent central que marginal (fig. : $g, j, k$ ) ; le noyau est sphérique ou élargi transversalement, la membrane ondulante inapparente. Ces Trypanosomes sont semblables à ceux désignés en 1912 par LAVERAN et MESNIL (11) sous le nom de T. montgomeryi mais dont la réalité spécifique est contestable (10). Nous considérons ces formes comme des variations dans l'espèce $T$. congolense.

Beaucoup de formes courtes présentent un kinétoplaste punctiforme et central (fig. : v, z); quelques unes des grandes formes aussi (fig. : $u$ ). Chez ces dernières, la membrane ondulante accuse genéralement un ou deux festons très nets (fig. : $a, p, w$ ). Dans sa grande majorité, cependant, la population est conforme à la description classique de $T$. congolense (fig. : $a, b, c, d . .$. ). 


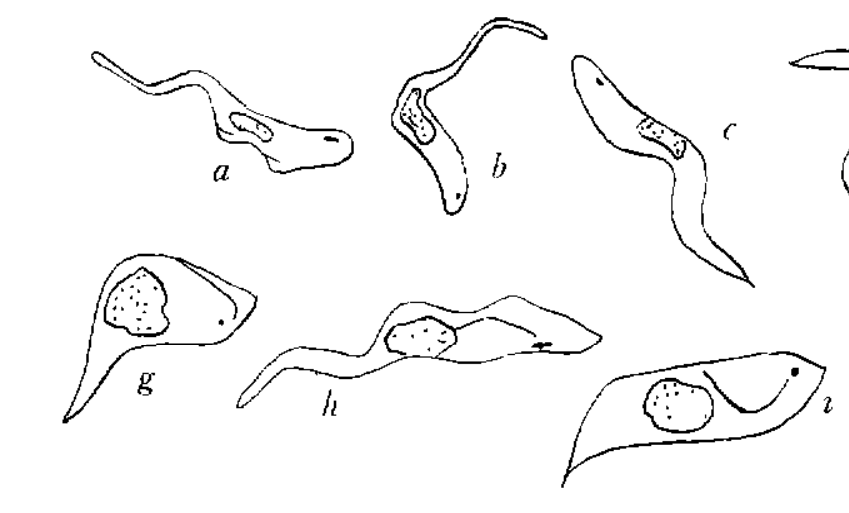

$\stackrel{10 \mu \mathrm{m}}{\longrightarrow}$

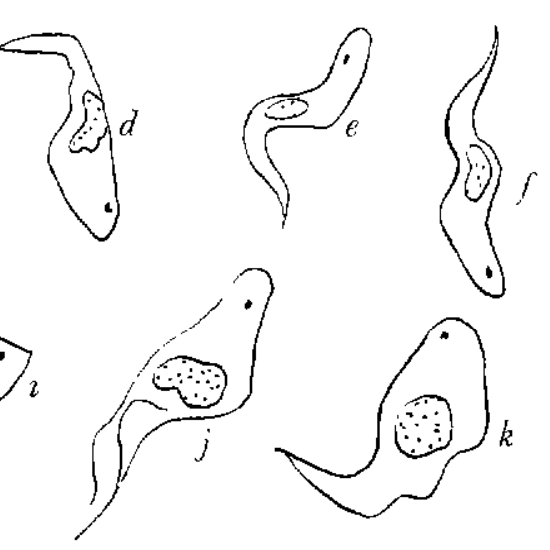

souche $C_{2} T$

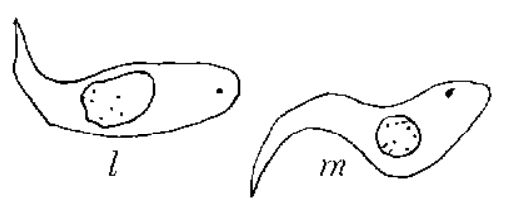

$\longrightarrow$
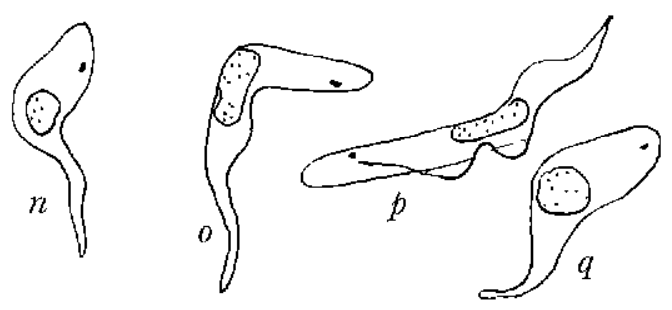

$C_{2} Q$

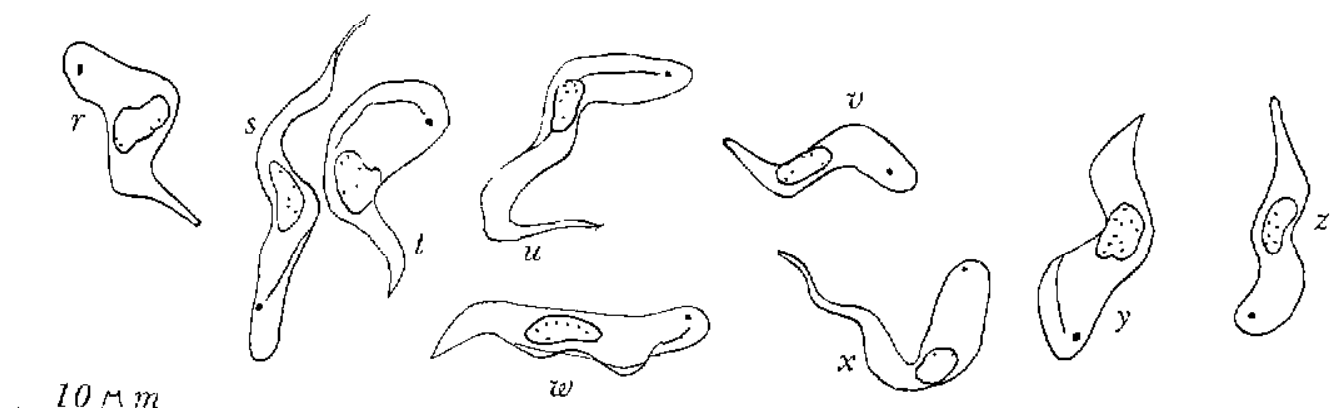

$C_{Q} T Q$
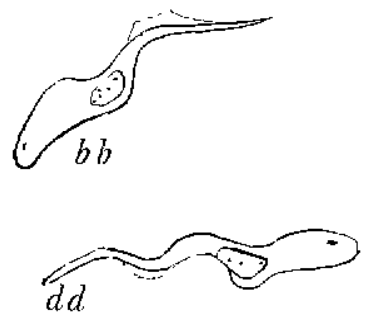

$10 \mathrm{Mm}$
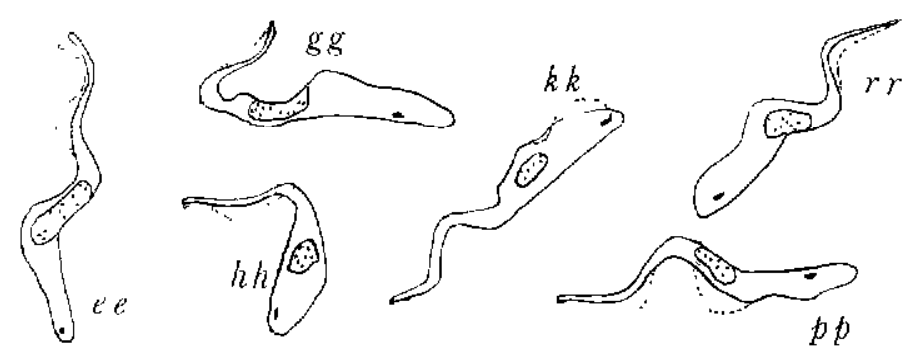

Variation morphologique de $T$. congolense .

de a à $\mathrm{z}$ : premiers passages sur souris ; de bb à pp : passages consécutifs. 
Les passages successifs chez la souris ont entraîné une diminution du nombre des formes massives. De plus, les individus chez lesquels apparaît une membrane ondulante sous forme d'l ou 2 festons sont plus nombreux que dans le premier passage. Enfin, on note un léger changement au niveau de l'extrémité antérieure du Trypanosome dont le flagelle se trouve bordé par une coulée pelliculaire de plus grande netteté que dans les premiers passages, réalisant ainsi une extrémité antérieure palmée (fig. : ee, $g g$, $p p, r r \ldots)$.

Pour ce qui est des passages très élevés, les Trypanosomes, quoique devenus beaucoup plus longs, gardent cependant la morphologie spécifique de $T$. congolense. On constate seulement une augmentation du nombre des organites intracytoplasmiques, à coloration métachromatique. Des études faites au microscope électronique sur la couche $C_{3}$ TQ au $157^{\circ}$ passage et au-delà font ressortir la présence de plages de sécrétions élaborées par des formations lamellaires ou recticulum sécrétoire $(2,14)$ et ce sont sans doute ces formations qui prennent une coloration métachromatique en microscopie photonique.

\section{OBSERVATIONS SUR LA VIRULENCE}

Les analyses faites sur les souris inoculées montrent dans les premiers passages des différences de comportement d'une souris à l'autre. Ces différences portent sur la durée de l'incubation et celle de la survie après l'apparition des premiers Trypanosomes dans le sang. L'incubation est de 8 à 12 jours pour le $1^{\text {er }}$ passage et elle diminue au cours des passages consécutifs : la durée de survie est de 15 à 30 jours et elle diminue aussi lors des passages. Chez quelques souris inoculées, l'acrné de l'infection est suivie d'une diminution considérable du nombre de Trypanosomes, voire de leur disparition totale. Dans un de ces cas, les Trypanosomes restants présentaient une grosse vacuole juxtanucléaire. Après diminution, les Trypanosomes se multiplient à nouveau au bout de 24 à $72 \mathrm{~h}$.

Dans les passages élevés, il a été constaté concernant la souche $C_{2} T$ une stabilisation de la virulence à l'égard des souris: du $472^{\mathrm{e}}$ au $572^{e}$ passage, sur 200 observations de souris, on note une durée moyenne de la période d'incubation égale à 3,31 jours $\pm 0,08$ (amplitude de 2 à 8 jours) et une durée moyenne de survie après l'inoculation égale à 9,18 jours $\pm 0,12$ (amplitude de 2 à 14 jours).

Le fait le plus important est la constatation que les passages élevés ne semblent pas atténuer le pouvoir infectant à l'égard du bovin, hôte d'origine de la souche $C_{2} T$. Deux bovins, inoculés avec cette souche au $581^{\mathrm{e}}$ passage sur souris, soit plus de dix ans après la récolte, sont devenus positifs en 5 jours et la parasitémie a persisté pendant un mois, délai au bout duquel les animaux ont été traités par l'Isométamidium.

Un autre bovin inoculé a une forte parasitémie au $8^{\circ}$ jour. Enfin deux Zébus, provenant d'une région indemne de trypanosomiase, sont positifs 5 jours après inoculation de Trypanosomes au $684^{\circ}$ passage. Tous ces animaux ont présenté, au cours des épreuves, une parasitémie élevée et des signes morbides qui attestent la persistance de la virulence de cette souche pour le bovin et confirment le type Dimorphon de l'espèce.

Une autre souche de $T$. congolense, non considérée dans ce rapport, $\mathrm{Z} 117$, a conduit à des observations similaires après inoculation à des bovins au $323^{\circ}$ passage. Les mêmes constatations se rapportent aussi aux souches de Trypanosoma brucei brucei après plus de 600 passages.

\section{DISCUSSION ET CONCLUSIONS}

1. L'analyse biométrique et l'étude morphologique de la variation autorisent à considérer les souches récoltées comme étant Trypanosoma (Nannomonas) congolense BRODEN, 1904. La longueur moyenne des populations mesurées oscille entre $13,34 \mu \mathrm{m}$ et $14,26 \mu \mathrm{m}$. Ces valeurs sont conformes à celles retenues pour $T$. congolense par plusieurs auteurs: LAVERAN et MESNIL (11), BAKER (1), HOARE $(7,8,9,10)$, GODFREY (4, 5, 6), FAIRBAIRN (3), MACKENZIE et BOYT (12).

Les populations comportent des individus de forme massive, semblables à ceux désignés sous le nom de Trypanosoma montgomeryi, cité par LAVERAN et MESNIL dans leur ouvrage de 1912, mais nous n'en reconnaissons pas l'indépendance spécifique tout comme STEPHEN, 1963 (13).

2. Au cours des passages, on assiste à un allongement de la longueur des Trypanosomes dans une même souche. Ce fait plaide en faveur 
de l'invalidation de Trypanosoma dimorphon en tant que taxon spécifique pour ne l'admettre que comme variation de $T$. congolense.

3. Il apparaît que les Trypanosomes d'une même souche peuvent se présenter, à un moment donné et à travers le temps, sous des aspects variables. Nous pensons qu'il convient d'éviter l'emploi d'expressions telles que : variétés, races, types, etc..., pouvant faire penser à des entités infraspécifiques immuables. S'agissant plus particulièrement de Trypanosoma congolense, nous estimons pouvoir éviter ces expressions en carac- térisant les formes observées par les qualitatifs : pachymorphes, leptomorphes, isomorphes ou nannomorphes pour désigner respectivement la variation montgomeryi, la variation Dimorphon et les formes caractéristiques normales de $T$. congolense.

4. Le fait de réaliser des passages élevés sur souris, mềme pendant dix ans, n'entraîne pas une perte de virulence de $T$. congolense à l'égard des bovins et il semble dès lors illusoire d'obtenir, par le procédé des passages, des souches utilisables pour vacciner.

\section{SUMMARY}

\section{Biometry, morphology and virulence of Trypanosoma (Nannomonas) Congolense during 640 passages into mice during 10 years}

Three strains of Trypanosoma congolense were ssolated in 1964 in the south-western Senegal and one of them passaged on mice as Iong as 10 years. The strains presented Dimorphon variation. During the passages, it was observed a lengthening of the Trypanosomes. High passages into mice, even practiced beyond 10 years, did not induce the lack of virulence of $T$. congolense for cattle and it seems illusory to obtain by the practice of passaces, strains which can be used for vaccine.

\section{RESUMEN}

Biometria, morfologia y virulencia de Trypanosoma (Nannomonas) congolense durante 640 pasajes sobre ratoncs durante diez años

En 1964, se recogieron tres cepas de Trypanosoma congolense en el surreste de Senegal y se ha pasado una de ellas sobre ratones durante más de diez años. Las tres cepas presentan la variación Dimorphon. Durante los pasajes sobre ratones, la largura de los tripanosomos aumenta.

Efectuar numerosos pasajes sobre ratones, además durante diez años, no tıene por consecuencia una pérdida de virulencia de $T$. congolense para con los bovinos y parece ılusorio obtener, mediante pasajes, cepas utilizables para vacunar.

\section{BIBLIOGRAPHIE}

1. BAKER (J. R.). Measurement of trypanosomes of the $T$. congolense group. E. Afr. vet. Res. Org., Report, 1956-1957, p. 28.

2. BOISSON (M. E.), BOISSON (C.) et MATTEI (X.). Présence d'un appareil élaborateur singulier chez. Trypanosoma congolense. C. R. Soc. Biol., Paris, 1967, $161(8-9)$ : $1772-1774$.

3. FAIRBAIRN (H.). Measurements of strains of Trypanosoma congolense. Ann. trop. Med. Parasit., 1962, 56 (2) : 218-221.

4. GODFREY (D. G.). Cyclical transmission of Trypanosoma dimorphon (?) by $G$. morsitans. West Afr. Inst. Tryp. Res., Report 1957, p. 21.

5. GODFREY (D. G.). Types of Trypanosoma congolense. I - Morphological differences. Ann. trop. Med. Parasit., 1960, 54 : 428-438.

6. GODFREY (D. G.). Types of Trypanosoma congolense. II - Differences in the courses of infection. Ann. trop. Med Parasit., 1961, 55 : 154.

7. HOARE (C. A.). (Révision de la classification des Trypanosomes pathogènes africains). Comité scient. int. Rech. Trypanosomiases, $6^{\mathrm{e}}$ réunion, Salisbury, $1956,67-80$.
8. HOARE (C. A.). Morphological and taxonomic studies on mammalian trypanosomes. IX. Revision of Trypanosoma dimorphon. Parasitology, 1959, 49 : 210-231.

9. HOARE (C. A.). Morphologıcal and taxonomic studies on mammalian trypanosomes. X. Revision of systematics. J. Protozool., 1964, 11 (2) : 200-207.

10. HOARE (C. A.). The Trypanosomes of mammals. Oxford and Edinburgh, Backwell Scientific Publ., 1972, 749 p.

11. LAVERAN (A.) et MESNIL (F.). Trypanosomes et trypanosomiases. $2^{\mathrm{e}}$ éd., Paris, Masson et Cie, $1912,1000 \mathrm{p}$.

12. MACKENZIE (P. K. I) et BOYT (W. P.). Notes upon a trypanosome strain ressembling $T$. congolense apparently completely adapted to the porcine species. Brit. vet. J., 1969, 125 (8) : 414-421.

13. STEPHEN (L. E.). On the validity of Trypanosoma montgomeryi, LAVERAN 1909. Ann. trop. Med. Parasit., 1963, 57 (4) : 397-403.

14. TOURE (S. M.). Etude ultrastructurale des effets produits par diverses drogues trypanocides sur les Trypanosomes. Rev. Elev. Méd. vét. Pays trop. 1971, 24 (3) : 381-392. 